\title{
Principales factores que conducen a una experiencia memorable para el viajero
}

\section{Main factors that lead to a memorable experience for the traveler}

\author{
Claudia E. Llontop Diez* \\ Escuela Profesional de Turismo y Hotelería, \\ Universidad de San Martín de Porres, Perú
}

\section{Resumen}

Respecto a las experiencias turísticas encontramos que para ser memorables es necesario tener en cuenta una serie de consideraciones que van desde los aspectos afectivos, cognitivos y de comportamiento humano, hasta una adecuada gestión de indicadores y uso de la tecnología.

Resaltamos la importancia del Journey map o analítica del recorrido del viaje, las distintas fases y puntos de contacto por las que atraviesa el visitante y su relación con factores que lo conducen a una experiencia memorable.

En ese sentido, la finalidad del presente trabajo es brindar a los responsables del diseño de destinos turísticos una serie de premisas teóricas y metodológicas para tomar en cuenta a la hora de emprender experiencias memorables para el viajero.

Palabras clave: experiencia turística, experiencia memorable, Journey map, puntos de contacto, diseño de experiencia. 


\begin{abstract}
Regarding tourism experiences, we believe that, if we want them to be memorable, it is important to take into account a series of considerations that cover from the affective and cognitive areas to the traveler's behavior, which must be supported by an adequate management of indicators and the use of technology.
\end{abstract}

We highlight the importance of the journey map or journey analytics, the contact points, and the different phases travelers go through, in order to assess their level of satisfaction and its relationship with factors that lead to a memorable experience.

In this sense, the purpose of this research is to provide those responsible for the design of tourist destinations that work on products under the umbrella of experiences with a series of theoretical and methodological premises to be taken into account at the moment of undertaking memorable experiences for the traveler.

Keywords: tourist experience, memorable experience, journey map, contact points, experience design.

\title{
La experiencia turística
}

La experiencia turística ha tenido un singular recorrido desde la teoría de la economía de la experiencia hasta nuestros días, y ha involucrado a muchos actores y académicos en los últimos años. En la actualidad, las empresas que apuntan a tener un lugar en el mercado con eficiencia, buscan integrar un diseño en función al cliente dentro de su plan de negocio. Es así que en los últimos años el concepto tiene una descripción multidimensional por parte de los investigadores. Cohen (1979), describe el concepto de experiencia de turismo como la relación entre las personas y su visión del mundo total, dependiendo de la ubicación de su centro con respecto a la sociedad a la que pertenecen (como se citó en Tung \& Ritchie, 2011).

Una interpretación quizá muy general, pero que engloba el concepto multidimensional de la experiencia, y evidencia la importancia de las 
relaciones personales en la experiencia turística, encontrando en este contexto un reto para descubrir aquello que en esencia hace a ciertas experiencias especiales, capaces de asombrarnos, de carácter espectacular, que quedan grabadas en nuestras vidas, en todo sentido memorable.

Tung y Ritchie (2011), se refieren a este concepto de dimensiones personales y emocionales señalando que las experiencias turísticas son:

La evaluación subjetiva de un individuo y la experiencia afectiva, cognitiva y de comportamiento de eventos relacionados con su actividad turística que comienza antes, es decir a partir de la planificación y preparación, durante, es decir, en el destino y después del viaje, es decir, el recuerdo. (p. 1369)

En este punto, como muchos investigadores en este campo de la experiencia, encuentran una solución a la evaluación subjetiva del visitante, empleando indicadores de satisfacción basados en dimensiones psicológicas de percepción (Tung \& Ritchie, 2011).

Lo más técnico para entender este complejo proceso es emplear una forma de observación ordenada y clasificada para su análisis, utilizando una herramienta que permita graficar el recorrido de la experiencia del cliente, esta herramienta es conocida como el Journey map, que permite comprender de manera organizada las numerosas fases o puntos de contacto que tiene el turista en una visita. Facilita la comprensión de las múltiples operaciones en el servicio de viajes turísticos y su relación con el turista.

El turismo es una industria de servicio intensivo que se centra en las experiencias de servicio de los clientes no solo durante su estadía, sino también antes y después de ella. Dado que la mayoría de los productos turísticos se reservan y pagan por adelantado, los clientes deben confiar en la precisión de la información accesible. (Stickdorn \& Zehrer, 2009, p. 2)

Moreno, Los Santos y Pascual (2017) adoptan como base en sus estudios las etapas del viaje propuestas por Google (2012), donde se divide el viaje en cinco fases: inspiración, planificación, reserva, viaje y compartir, incorporando además, los nuevos medios digitales utilizados para la gestión del viaje. 


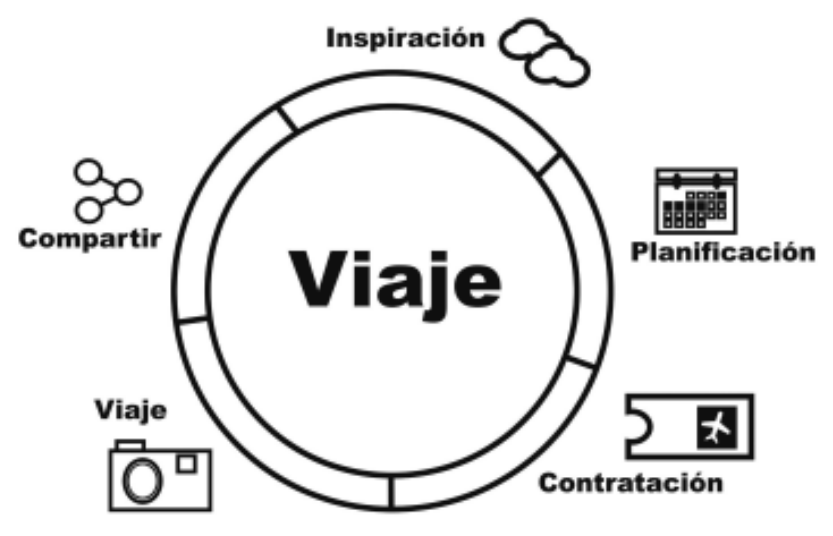

Figura 1. Interpretación gráfica de las fases del viaje de Google (2012). Adaptado de Moreno et al., 2017, p. 304.

La importancia de esta desagregación de las fases del viaje y la incorporación de internet en todo el ciclo del viaje se pone de manifiesto en los indicadores aportados por Google.

\section{Encontrando las dimensiones humanas del nuevo turista}

\section{Conectando la experiencia memorable}

En los últimos 30 años se han generado cambios tecnológicos y una transformación en las formas y medios de comunicación, situación que ha provocado un giro en todas las actividades, de manera particular en el sector turismo. En el turismo el cambio es constante, reconocemos que este lleva una esencia o algo fundamental que lo relaciona al hombre y la posibilidad de ver, experimentar, sentir, y además, aprender algo para la vida, tal como lo señalan Brent, Tung y Ritchie (2011):

La esencia del turismo en el mundo de hoy es el desarrollo y la entrega de experiencias de viaje y visitas a una variedad de individuos y grupos que desean ver, comprender y experimentar la naturaleza de diferentes destinos y la forma en que la gente vive, trabaja y disfruta de la vida en esos destinos. (p. 419) 
Las capacidades generacionales son más marcadas, unas de otras como resultado de cambios tecnológicos, la conectividad y la comunicación; como consecuencia de ello existe una tendencia al cambio, a la manera de actuar e interactuar con las personas, respecto al turismo, lo que es importante reconocer.

Desde el punto de vista de la demanda, el nuevo turista está cada vez más informado, es más sensible a la calidad y está más dispuesto a reaccionar rápidamente ante los cambios en el mercado turístico después de publicaciones en sitios web de calificación turística. Por lo tanto, la calidad percibida del producto de servicio emerge como el factor crucial en la fase de pre-compra de los productos turísticos. (Stickdorn \& Zehrer, 2009, p. 12)

Hay un nuevo turista que en ciertos términos es informado, sensible a la calidad y tiene la facilidad de usar medios de comunicación para expresarse, que tiene la capacidad de compartir (share), recibe y evalúa servicios, reconoce el servicio como un todo en general, las fallas o decepciones que pueden surgir.

El concepto de puntos de contacto sirve para establecer posibles errores en la entrega del servicio, diagnosticando en qué parte del circuito se cometió el error, sin embargo entendiendo las características del servicio, debemos tener claro que el cliente no entiende de instancias administrativas y evalúa el servicio como un todo integrado.

Los consumidores esperan que las marcas conozcan intuitivamente lo que necesitan, cuándo lo necesitan, por qué lo necesitan y además que lo entreguen al instante, en el momento que lo necesitan. A medida que la tecnología cambia, también lo hacen las expectativas del consumidor. Los clientes de hoy son impacientes y sus estándares son implacables (Maloney, 2017).

Una característica personal de esta condición otorgada por la conectividad y el uso de teléfonos móviles es la influencia de la velocidad en el cliente, al punto de convertirlo en impaciente, gran reto para los profesionales del marketing, quienes buscan conocer a los clientes y sus necesidades. 
En el artículo de Matt Lawson vicepresidente, Ads Marketing en Google citando a Laura Beaudin, socia de Bain \& Company, afirma que ya no es suficiente saber quién es el cliente. Nos explica que estamos entrando en una era en la que ser relevante para esos clientes significa construir estrategias en torno al marketing oportuno para garantizar que les esté llegando cuando estén listos para participar (como se citó en Lawson, 2018).

Es importante conocer al cliente, pero encontramos que en estos tiempos es más importante ser relevantes, y ello no solo implica llamar su atención, significa estar presente o mejor dicho estar en la conciencia del cliente y que este tenga la certeza que estaremos ahí para asistirlo siempre que nos necesite, ese es el verdadero interés del cliente de hoy, sobre todo en el sector turismo.

El viaje está compuesto por elementos que pueden estar al alcance del visitante y que puede utilizar para enriquecer su experiencia. La presencia inadvertida de aspectos invisibles $u$ ocultos en las operaciones puede derivar al éxito o fracaso de las mismas. Por lo tanto, la presencia de herramientas en forma latente durante el viaje se torna en un reto para los diseñadores de experiencias memorables.

La tecnología hace más fácil esta presencia, pues en muchos lugares turísticos se colocan códigos Qr con la finalidad de conectar al visitante con información relevante del lugar o monumento que visita, aunque no todos acceden al enlace, el código está presente en lugares estratégicos a la espera de ser útil al visitante.

El turista que viaja a un destino turístico no realiza meramente un viaje físico. El verdadero viaje es interior, y radica en cómo percibe el lugar que visita, sus gentes, y el impacto que esta percepción subjetiva y personal le produce. (Carballo, Moreno, León, \& Brent, 2015, p. 72)

Este enfoque que prioriza la importancia del visitante como protagonista en la experiencia es sumamente importante, enfoca al turista como persona, antes que cliente, y hace más evidente la necesidad de mirar en su interior de una manera más íntima. La correspondencia de sus expectativas, anhelos y sentimientos con las interacciones que realiza en contacto con otras personas durante una visita desencadena en una experiencia memorable. 
Bajo esta mirada, el cliente no es una simple cuenta que genera ingresos, una cifra, o base de datos, al mirar al visitante desde esta perspectiva, encontramos que la experiencia tiende a ser memorable cuando involucra relaciones de persona a persona, en donde el visitante puede encontrar correspondencia a lo que busca o sorprenderse al encontrar algo diferente que le otorgue un sentido de novedad a la experiencia.

En este sentido, Chandralal y Valenzuela (2013) afirman que por lo general hay una mayor probabilidad de que un evento se vuelva memorable debido a las personas, a los eventos sociales más que al lugar en sí mismo. Encuentran, en esta relación entre personas, un hallazgo de suma importancia para la comprensión de una experiencia memorable, que está relacionada con dar significado a la misma experiencia, y que se clasifica en: autodesarrollo, desarrollo de relaciones y mejora del bienestar familiar, como sentido a lo experimentado. El desarrollo de las relaciones es el aspecto más importante del viaje, que incluye el fortalecimiento de los vínculos existentes con los integrantes del viaje, por ejemplo, el desarrollo de nuevas amistades con otros viajeros y gente de la localidad.

Pero en la actualidad, no solo encontramos formas presenciales para relacionarnos con los demás -sobre todo si estamos en lugares alejados del entorno familiar o amical, que es cuando vemos la necesidad de comunicarnos o estar presente en la memoria de ellos-, también utilizamos la tecnología para acercarnos, comunicarnos con los demás, empleando las bondades de la conectividad móvil, plataformas sociales y de comunicación en línea, haciendo llamadas, videollamadas, fotos, audios y otras formas de interacción en el sitio que visitamos; allí también encontramos un vínculo y un sentido en tiempo real con comunicación a distancia.

Este tipo de interacción con la experiencia, la podemos encontrar tanto en la fase de búsqueda como en la fase final del viaje, sin embargo, existe una fase concreta, la fase de compartir, que se inicia en el preciso momento en el que comentas o socializas tus experiencias con amigos, o con alguna comunidad en las redes a manera de recomendación. La relación será siempre con otras personas, lo que cambia es el medio en el que se comunica. 
En este sentido, los sitios web de calificación de clientes se vuelven cada vez más populares, ofrecen a los clientes acceso abierto a revisiones de clientes en paralelo con una comparación de precios en tiempo real, con respecto al transporte, alojamiento o actividades recreativas y de ocio. (Stickdorn \& Zehrer, 2009, p. 2)

Esta nueva forma de acercar a las personas, crea una conexión haciendo posible, a pesar de las distancias, interactuar, y generar vínculos, influyendo en los aspectos afectivo, cognitivo y de comportamiento en el visitante.

\section{Diseño y experiencia del cliente}

En los últimos años, la preocupación por otorgar una adecuada experiencia al visitante se ha convertido en la misión más importante para muchos emprendimientos que buscan mejorar la eficiencia de la empresa en el competitivo mercado del sector turismo. Stickdorn y Zehrer (2009) afirman que:

La competitividad de cualquier producto de servicio depende en última instancia de la satisfacción del cliente, y que está determinada por la evaluación del consumidor de las expectativas hacia un determinado producto y las experiencias reales con el proceso de entrega del producto. (p. 1)

Una adecuada experiencia de compra trae consigo un nivel alto de satisfacción, la clave de la experiencia del cliente está en la innovación y diseños creativos que no solo requieren de una gran capacidad de abstracción, sino también de la habilidad de conocer y entender al cliente (Richardson, 2010a). En tal sentido Verhoef et al. (2009) plantean:

La creación de una experiencia de cliente superior parece ser uno de los objetivos centrales en los entornos minoristas actuales. Minoristas que, en todo el mundo han adoptado el concepto de gestión de la experiencia del cliente, y muchos han incorporado la noción en sus declaraciones de misión. (p. 1)

$\mathrm{Al}$ respecto, Zehrer (2009, p. 337) considera los servicios como productos, que deben ser desarrollados sistemáticamente con un claro enfoque en el valor del 
cliente, evidenciando que no solo se debe identificar cada punto de contacto, si no también asegurar la entrega de valor en estos. La búsqueda de diseños e ideas innovadoras se debe enfocar en crear y desarrollar nuevas formas para que la entrega del servicio se convierta en experiencia memorable, pues ello implica una transformación en la cultura de la empresa y sus procesos.

En contraposición a esta abierta necesidad de entender la experiencia del cliente encontramos que, las investigaciones centradas en la experiencia del cliente aún no son suficientes y su difusión es limitada. «Solo un número limitado de artículos exploran la experiencia del cliente en profundidad desde una perspectiva teórica» (Verhoef et al., 2009, p. 31).

Aquellos servicios que no integran experiencias en su diseño, poco pueden esperar de ser recordadas. Un diseño mal concebido no le ofrece a los clientes una clara percepción de sus experiencias, impidiendo ordenar en su mente un registro de impresiones, en consecuencia, es menos probable que la experiencia permanezca en la memoria (Pine \& Gilmore, 1998).

El diseño de la experiencia del cliente en turismo debe contemplar una propuesta de entrega de servicio, que responda no solo a los requerimientos básicos de calidad para cubrir las expectativas del cliente, sino además debe enriquecerse con la información obtenida del feedback o retroalimentación del mismo cliente y la de otros usuarios. Aunque llevar el diseño a la acción se hace evidente como tarea, su ejecución es un tanto compleja y difícil de aplicar, mucho más complicado es lograr que funcione como un constructo subjetivo y teórico, e integrarlo a los procesos de la empresa. En este sentido, para Richardson (2010b) «las experiencias del cliente surgen de elementos concretos y controlables: los puntos de contacto» (p. 2).

La clave está en identificar y codificar estos puntos de contacto, es tarea de la empresa trabajarlos e integrarlos, una vez que son identificados, dejando atrás especulaciones o conceptos etéreos que no son medibles o factibles de analizar (Richardson, 2010a).

Se puede argumentar que las empresas no pueden controlar completamente las experiencias, porque las experiencias inevitablemente implican percepción, emoción y comportamientos inesperados en las partes 
de los clientes. La gente no se comporta como un robot, y no importa qué tan bien elaboramos una experiencia, no percibirán exactamente como anticipamos o esperamos (Richardson, 2010b, p. 1).

Lo complejo de esta situación, del control y la responsabilidad de la empresa por crear una experiencia, nos atrapa en un círculo de indecisión y de alguna manera la situación se torna intimidante e inalcanzable. Para comprender un poco más de la experiencia del cliente debemos entender que, en la vida cotidiana, las empresas desarrollan un tipo de experiencia en sus clientes, sin embargo, la empresa muchas veces no conoce cómo es, ni qué elementos intervienen; pueden intuir por sus resultados y la observación simple de sus clientes que están haciendo algo bueno o malo. Pero esto no basta, «esa experiencia puede ser buena, mala o indiferente, pero el solo hecho de que tengas clientes, interactúes con ellos y les proporciones productos y servicios, significa que tienen una experiencia contigo y tú marca» (Richardson, 2010b, p. 1).

De este modo, la comprensión de las necesidades del cliente y la identificación de los puntos de contacto hacen posible en el tiempo que una experiencia se convierta en experiencia memorable. En este sentido la tarea es de largo aliento, de mucha observación y capacidad de cambio en la gestión de la empresa para intervenir de manera efectiva en las diversas interacciones o puntos de contacto con el cliente. En este mismo sentido, Stickdorn y Zehrer (2009) afirman:

El punto inicial del diseño del servicio es el cliente y, por lo tanto, es esencial recopilar un conocimiento adecuado sobre su motivación de viaje y aprender más acerca de qué servicios constituye su respectivo paquete de productos y qué productos de servicio son factores clave para el éxito o el fracaso de los productos turísticos. (p. 6)

Las múltiples fases del servicio son una fuente rica en oportunidades para interactuar con el cliente otorgándole experiencias (Stickdorn \& Zehrer, 2009). «La experiencia del cliente abarca la experiencia total, incluidas las fases de búsqueda, compra, consumo y posventa de la experiencia, y puede implicar múltiples canales minoristas» (Verhoef et al., 2009, p. 46). 
No hay medias tintas para abordar una política centrada en la experiencia del cliente, si la empresa se enfoca, es posible que en el proceso pueda tener errores, pero estará camino a una gestión de la experiencia del cliente, el lado opuesto a esta actitud es la de no mirar estas interacciones y no generar indicadores para evaluar la gestión, es simplemente no actuar o manejar sin ver el camino.

Richardson (2010a), con respecto a la experiencia del cliente, sostiene que «es la suma total de cómo los clientes interactúan con su empresa y marca, no solo en una instantánea en el tiempo, sino a lo largo de todo el proceso de ser un cliente» (p. 1).

De modo que, encontramos una grata coincidencia en los diseños turísticos, con vocación por entregar siempre una propuesta de valor al visitante y su preocupación por las experiencias turísticas.

El diseño de los servicios con miras a crear experiencias memorables y satisfactorias para el cliente no es nuevo; sin embargo, se puede decir que el diseño deliberado y la ejecución de experiencias de servicio como una disciplina de gestión distintiva con sus propios principios, herramientas y técnicas son un nuevo enfoque. (Zehrer, 2009, p. 333)

La diferencia está en la acción y la capacidad de ejecución, todo lo explicado quizá suene muy inspirador y corresponda a los anhelos de todo emprendedor, pero aquello que lo diferencia está en un diseño con capacidad de mostrar indicadores empleando conectividad, uso de aplicaciones web y herramientas tecnológicas que hagan posible su integración a la gestión de la empresa de una manera eficiente y organizada para la toma de decisiones (Zehrer, 2009).

\section{Journey map y los puntos de contacto con el cliente}

Entendida la estructura y el valor del diseño, debemos aclarar que todo diseño de producto se realiza en función al usuario, por lo tanto, es importante tener en cuenta que las preferencias se pueden agrupar en segmentos, grupos de personas, necesidades y motivaciones similares. 
Stickdorn y Zehrer (2009) coinciden con otros autores al referir que, para conocer los segmentos se deben analizar rasgos, estereotipos y condiciones que permitan diferenciarlos de otros, analizando también sus similitudes para agruparlos, basados en trabajos de campo de corte etnográfico, cuantitativo o mixto, para identificar grupos comunes.

En cuanto a los puntos de contacto con el cliente, el siguiente paso está relacionado a cómo intervenir con el momento de la verdad tal como lo refiere, o los momentos en el que el cliente genera una interacción con el servicio (Grönroos, 1990). Un punto de contacto es cualquier elemento físico o digital del sistema con el que el usuario entra en contacto durante la experiencia. Puede ser un dispositivo de hardware, una aplicación de software, un servicio web e incluso un examen físico, espacio o herramienta (Brugnoli, 2009).

De este modo, los puntos de contacto se multiplican al analizar nuestro modelo de servicio, añadiéndose también una cantidad de variables e indicadores para analizar e interpretar. Una forma ordenada y organizada, empleada en la comprensión de estos puntos de contacto es el Journey Map o mapa de recorrido del cliente.

Un mapa de viaje del cliente es una idea muy simple: un diagrama que ilustra los pasos que su(s) cliente(s) realiza(n) al entrar en contacto con su compañía, ya sea este un producto, una experiencia en línea, una experiencia minorista, un servicio suelto o combinación de servicios. Mientras más puntos de referencia se contemplen en el mapa, más complicado será el análisis, pues es importante que el mapa de viaje del cliente refleje todo el proceso de inicio a fin o como popularmente lo llaman «de la cuna a la tumba». El mapa de viaje, es un cronograma del viaje del cliente que incluye primero entrar en contacto con un cliente (tal vez con publicidad o en una tienda), comprar el producto o servicio, usarlo, compartir la experiencia con otras personas (en persona o en línea) y luego terminar el viaje actualizando, reemplazando o eligiendo un competidor o reestableciendo el viaje con otra compañía (Richardson, 2010a).

Tener un mapa estructurado y ordenado nos facilita la comprensión del trayecto o ruta que experimenta el cliente al recibir el servicio, que trabajando sus variables e indicadores se puede convertir en una herramienta eficiente para la evaluación de los momentos que conforman la experiencia de viaje. 
En esta línea, un viaje al cliente incluye no solo puntos de contacto directos entre clientes y un proveedor de servicio respectivo, sino también indirectos, como los sitios web de revisión mencionados. De hecho, la mayoría de estos puntos de contacto indirectos constituyen el punto de partida de un viaje al cliente en el turismo atrayendo la atención de los turistas hacia un determinado destino, por ejemplo, sitios web de turismo, guías de turismo, diarios de viaje o 'boca a boca' (Stickdorn \& Zehrer, 2009).

La afirmación de Stickdorn y Zehrer (2009) sobre puntos de contacto indirectos se basa en la observación de la búsqueda en la primera fase del viaje donde el cliente no solo tiene contacto con la web o información del proveedor, también se asegura de una buena decisión con la información de otros recomendadores web o la misma opinión de foros, amigos o comunidad, es en este paso donde también se generan las expectativas en relación al servicio y la experiencia.

Cada interacción genera un vínculo y a la vez se convierte en argumento para evaluar o emitir un juicio, en este sentido podemos decir que en cada contacto siempre encontramos una retroalimentación y una oportunidad de aprendizaje. Se retroalimenta en la fase de 'sueño de viaje' según Google, o la fase de búsqueda para otros autores, se refuerza cuando existe una duda sobre algún aspecto del servicio, o cuando simplemente buscamos corroborar si está en lo cierto. Sucede cuando estamos en un restaurante y preguntamos si el plato que estamos degustando es delicioso, rápidamente alguien en la mesa lo corrobora y en algunos casos añade algo de sus experiencias pasadas para emitir un juicio, positivo, negativo o neutro. En tal sentido, «los clientes definen qué puntos de contacto son notables y cuáles ignoran o pasan por alto» (Stickdorn \& Zehrer, 2009, p. 7).

Claramente, se puede establecer que este punto de reflexión involucra el juicio del cliente para determinar la satisfacción o insatisfacción del servicio recibido y también la oportunidad de conocer aquellos aspectos positivos que pueden superar los negativos, en una evaluación holística de la experiencia.

\section{Satisfacción en la gestión de la experiencia}

La misión de toda empresa radica en brindar un buen servicio y un indicador de ello es la satisfacción, para ello es importante que toda empresa 
se comprometa a desarrollar una adecuada gestión de calidad en sus operaciones. Al respecto Zehrer (2009) afirma:

El turismo es una industria intensiva en servicios que depende de la calidad de las experiencias de servicio de los clientes y de sus evaluaciones consecuentes de satisfacción o insatisfacción. La gestión de la calidad del servicio es, por lo tanto, de crucial importancia para el turismo. (p. 332)

La satisfacción se confirma o des-confirma cada vez que el cliente experimenta el servicio, y su confirmación es el resultado de comparar lo experimentado con sus sentidos y sus expectativas. Este es el llamado paradigma de confirmación y des-confirmación. «Este modelo orientado a procesos de satisfacción del cliente se denomina paradigma de confirmación y des-confirmación, ampliamente aceptado como un enfoque aplicable para medir la satisfacción del cliente» (Stickdorn \& Zehrer, 2009, p. 6).

La vía que los investigadores han empleado en los últimos años para evaluar situaciones subjetivas y complejas en el servicio es el paradigma de confirmación y des-confirmación, que en el caso de las experiencias memorables engloba todos aquellos factores afectivos, cognitivos y de comportamiento, que al interactuar entre sí, bajo un enfoque sistémico nos puede llevar a conocer en profundidad aquellos aspectos más relevantes que motivan o dan como resultado una experiencia memorable.

Respecto a la satisfacción, Stickdorn y Zehrer (2009) encuentran variables que influyen en la satisfacción y las agrupan en factores básicos, factores de rendimiento y factores de entusiasmo para comprender de manera holística la calidad percibida, cada uno de ellos influye en la satisfacción general como resultado de una escala de niveles de expectativa. Además, sostienen que los factores básicos se caracterizan por un alto nivel de expectativa pero un bajo impacto en la satisfacción general. Por lo tanto, los clientes requieren de factores básicos y su ausencia o mal desempeño conducen a la insatisfacción, mientras que solo en casos excepcionales un logro superior de los factores básicos contribuye significativamente a la satisfacción general. El impacto de los factores de rendimiento en la satisfacción general es proporcional a sus expectativas $\mathrm{y}$, por lo tanto, puede clasificarse como poco importante e 
importante. Los factores de entusiasmo se definen por un nivel de expectativa bajo pero alto impacto en la satisfacción general. La presencia de estos factores genera satisfacción general del cliente, pero no pueden compensar los factores básicos incumplidos. Esta estructura nos puede orientar en la búsqueda de variables para analizar los puntos de contacto con el cliente y por lo tanto la evaluación del nivel de satisfacción percibida si se emplean los indicadores adecuados.

\section{Dimensiones y factores que influyen en la experiencia del cliente}

Integrar esta concepción de variables que influyen en la satisfacción por medio de estos factores en la experiencia memorable, nos lleva a reflexionar más detenidamente sobre cuáles son las dimensiones de la experiencia del cliente.

Pine y Gilmore (1998), en su teoría de la economía de la experiencia, identificaron cuatro ámbitos de experiencias del cliente: entretenimiento, estética, educación y escapismo. Estas cuatro dimensiones de la experiencia del cliente influyen en sus recuerdos y lealtad (Ali, Hussain, \& Ragavan, 2014). En consecuencia, estos autores amplían la comprensión de la economía de la experiencia enfocándola al turismo, examinando cómo funciona la memoria de los turistas junto con múltiples dimensiones. Para Hosany y Witham, «estas cuatro dimensiones se diferencian en dos ejes: el grado de implicación del cliente y la conexión del cliente con el entorno» (2010, p. 1).

Ali et al. (2014, p. 274), se refieren a las cuatro dimensiones de la experiencia que formulan Pine y Gilmore, señalando que la primera dimensión, el entretenimiento, se desarrolla cuando la absorción pasiva es observada por el cliente, como ver un espectáculo teatral; la segunda dimensión, la estética, donde el cliente tiene una participación pasiva e inmersión en la experiencia; la tercera dimensión, la educación, que se refiere a una experiencia en la que el participante participa activamente pero está en estado de absorción, como clases de buceo, baile, matricularse en una escuela de surf; la cuarta dimensión, el escapismo, que ocurre cuando el participante realiza actuaciones o actividades en tiempo real, en el entorno real o virtual, por ejemplo, haciendo rafting o jugando en un casino. En resumen, se puede afirmar que la experiencia de entretenimiento se trata de sentimientos, el aprendizaje de la experiencia educativa, la presencia de la experiencia estética y la experiencia 
escapista influenciando en las emociones de los clientes y niveles de satisfacción.

Hussain y Ragavan (2014) refieren que son pocos los estudios académicos en cuanto a la evaluación de la experiencia turística basada en los cuatro reinos conocidos como las 4E de Pine y Gilmore; sin embargo, hoy en día estas propuestas se toman como referencia en muchas investigaciones de campo.

Una perspectiva que describe un nivel de involucramiento o participación del visitante con respecto a la experiencia es la de Arnould y Price (1993) quienes describen tres dimensiones, en donde la palabra comunión la añaden para establecer un tipo de comunicación especial o vínculo, que los une con características especiales, para ellos, estas son tres dimensiones claves de una experiencia extraordinaria:

- Comunión con la naturaleza,

- Comunión con amigos, familia e incluso extraños,

- Crecimiento personal y renovación de uno mismo.

La propuesta de una conexión personal nace de la explicación de esta especial comunión en el plano personal y social involucrando el entorno donde se desarrolla la experiencia.

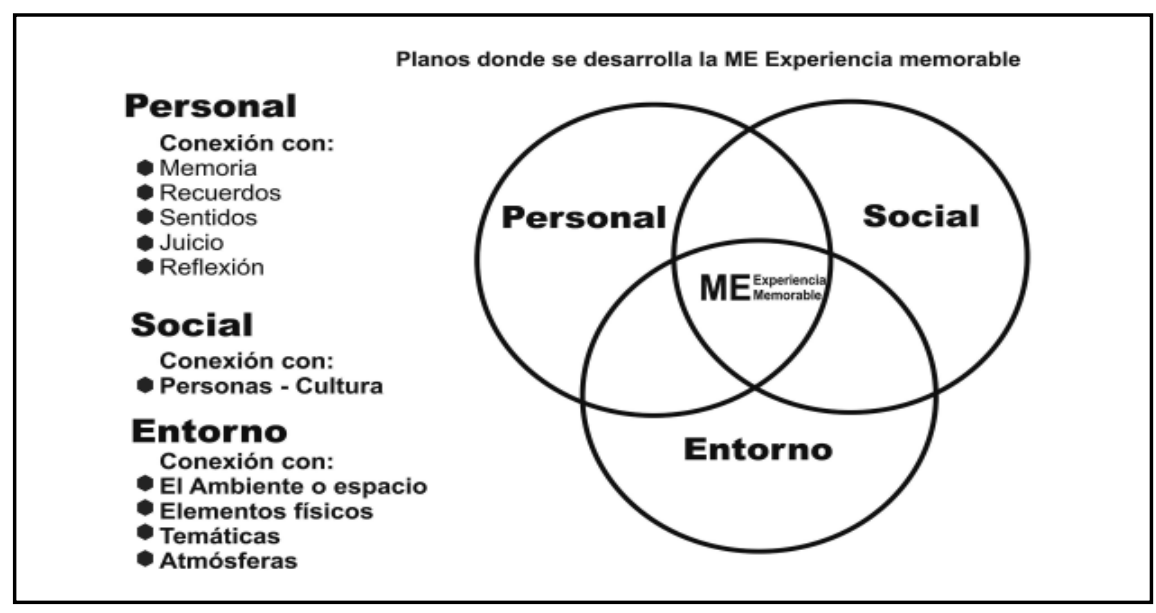

Figura 2. Interpretación de los planos donde se desarrolla la experiencia memorable. 
El entorno donde se desarrolla la experiencia, muestra tres planos que en su conjunto coinciden en una intersección llamada experiencia memorable. Estos planos corresponden al contexto personal que permite la conexión con la memoria, los recuerdos, los sentidos, etc., el contexto social que permite la conexión con las personas y la cultura del lugar anfitrión y el entorno que cuenta con una conexión con el espacio y la atmósfera.

En las interacciones donde se genera este vínculo de comunión se desarrolla una comunicación especial de empatía con un sentido de unión, por lo tanto, aquellas experiencias que se desenvuelven en esta intersección, tienen más posibilidad de permanecer en la memoria e influir en la percepción de la satisfacción (Arnould \& Price, 1993).

Las experiencias memorables parten de alguna manera del deseo, expresadas como una búsqueda que llena el espacio interior del hombre, ese conjunto de expectativas que van más allá de la primera fase del viaje que algunos consideran el motivo de soñar, de este modo, quien verdaderamente tiene una experiencia memorable trae consigo un conjunto de expectativas que despiertan ese deseo, tal como lo hacen en su vida diaria, en su búsqueda del sentido de la vida, como forma razonable de comprender lo experimentado.

Como podemos observar, cada investigador ha tomado un rumbo añadiendo más énfasis en determinadas dimensiones debido a la diversidad de la oferta turística y los múltiples servicios que ofrece. De acuerdo a su especialidad han planteado su punto de vista en cuanto a las dimensiones y variables que han empleado en sus trabajos de investigación.

A continuación se describen las dimensiones y aspectos más relevantes en cuanto a las experiencias memorables, de acuerdo a diferentes autores.

Kim, Ritchie y Cormick (2012) refieren en sus modelos conceptuales una serie de dimensiones constructivas diferentes, tales como hedonismo, paz mental, participación, reconocimiento, entretenimiento, estética y educación. Sin embargo, parecen haber descuidado la memoria, que es un factor importante en el desarrollo de modelos conceptuales de experiencias turísticas. 
Los trabajos de Ballantyne, Packer y Sutherland (2011) parten del estudio de las experiencias en áreas naturales silvestres buscando el cambio de actitud $\mathrm{y}$ aprendizaje incorporando aspectos cognitivos, afectivos y de comportamiento relacionados al aprendizaje.

Las dimensiones trabajadas son:

- Impresiones sensoriales. Los visitantes informan recuerdos visuales, auditivos, olfativos o táctiles de su experiencia.

- Afinidad emocional. Los visitantes informan respuestas emocionales a la experiencia o conexiones emocionales con los animales que observaron.

- Respuesta reflexiva. Los visitantes informan nuevas ideas como resultado del procesamiento cognitivo de su experiencia o hacen comentarios que indican que han reflexionado sobre lo que vieron o escucharon.

- Respuesta de comportamiento. Los visitantes informan haber tomado acciones específicas en respuesta a su experiencia de turismo de vida silvestre o informar una mayor conciencia de la necesidad de tal acción.

En la dimensión reflexiva aportan aquel ingrediente faltante en la experiencia y es la reacción del visitante o la respuesta a los estímulos desde la razón, denominada 'respuesta reflexiva' como un acto previo o consecuencia del comportamiento.

Tung y Ritchie (2011) presentan la investigación de Woodside, Caldwell y Albers-Miller, donde refieren que las experiencias memorables positivas de los turistas, han dado como resultado la posibilidad de volver a visitar un destino y difundir el «boca a boca positivo». Sin embargo, otros estudios en donde se aplica el factor novedad, indican que los turistas no retornarían al lugar visitado dos veces, es por ello, que en el caso de destinos esto solo aplica a todos aquellos relacionados a excursiones de fin de semana o de cierto tipo de vacaciones habituales.

Cada factor o dimensión corresponde a un tipo de viaje o experiencia de viaje, con alguna diferencia interpretativa de intensidad o variante, por ello los instrumentos deben contemplar todas las posibilidades y casos que se pueden presentar. 
El trabajo de Tung y Ritchie (2011) se basa en el estudio de:

- Experiencias satisfactorias

- Manejando experiencias memorables

- Recuerdos y experiencias

- Trabajo de memoria

- Mindlessness-Mindfullness

- Formación de memoria y retención

Identifica cuatro dimensiones que representan aspectos de experiencias que permiten ser particularmente memorables: afectividad, expectativas, consecuencialidad y recuerdo. En este sentido la investigación de Tung y Ritchie (2011) encontró que la percepción de un destino como «novedoso», está relacionado a las experiencias de aprendizaje percibidas como «frescas y reveladoras» por parte de los participantes en todos los aspectos del viaje (Tung \& Ritchie, 2011, p. 1380). Otros autores explican el proceso de inmersión en las experiencias como una característica distintiva de la experiencia turística memorable. «Ser uno con la experiencia ayuda al crecimiento y esto en sí mismo es un elemento extremadamente importante» (Horváth, 2013, p. 1). Este proceso de inmersión lo encontramos en el momento de contacto de la persona con la experiencia, que se activa ante un estado de conciencia. Al respecto, Pine y Gilmore (1998) describen en su propuesta de los 4E, conocida como los cuatro reinos del valor de la experiencia (Educación, Estética, Escapismo y Entretenimiento) que en el paso de transición entre las experiencias, encontramos un estadío o fase de participación activa o pasiva del cliente, absorción o inmersión de este en la experiencia.

La investigación de Kabat-Zinn (2003) sobre mindfulness y la de Tung y Ritchie (2011) resaltan la importancia de esta capacidad de inmersión en las experiencias por parte del participante para lograr un especial posicionamiento de la experiencia en su memoria. Al respecto, Kabat-Zinn (2003) afirma que una definición operacional de trabajo de mindfulness es «la conciencia que emerge prestando atención a propósito, en el momento presente, y sin prejuicios al desarrollo de la experiencia momento a momento» (p. 145). 
El proceso de inmersión de Horváth (2013) en la experiencia se retrata en muchas investigaciones como mindfulness y también su aspecto opuesto como mindlessness, en este contexto la atención prestada a la experiencia es producida por una actitud frente a la experiencia que se refleja en un acontecimiento memorable cuando este actúa. De este modo, cada momento puede convertirse en una experiencia memorable, el estado de mindfulness permite que las percepciones adquieran un significado y un sentido en la razón, en este punto la memoria almacena lo experimentado y lo confronta con la razón, evocando otros recuerdos. Es así que, en este ciclo el deseo recurre a la memoria para confrontar o encontrar correspondencia, iniciando un proceso de búsqueda interior que desencadena en este activador de la actitud turística.

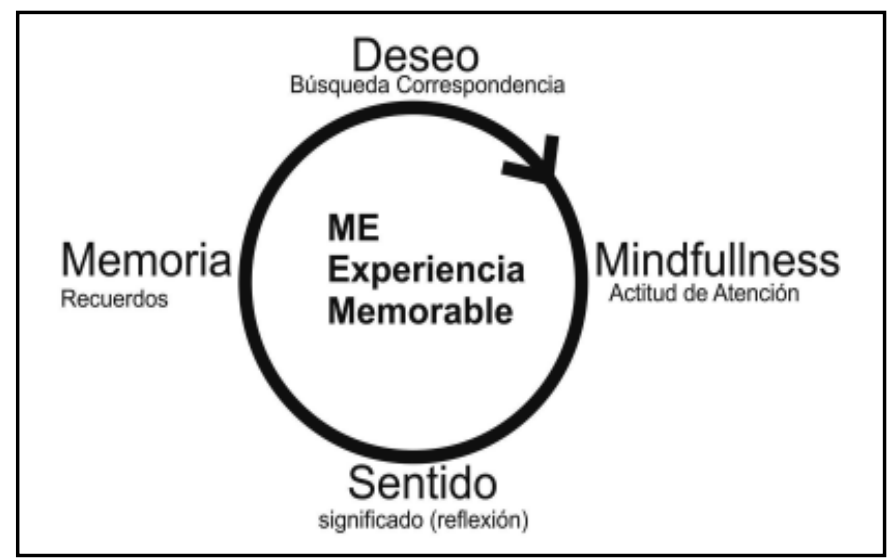

Figura 3. Fases por las que una experiencia se transforma en una experiencia memorable.

Encontramos también, que el aporte de Horváth (2013) está relacionado a la cocreación en el consumo de experiencias y resalta la importancia de las interacciones humanas como factor importante en la experiencia memorable, afirma que el objetivo de la creación de la experiencia turística es el individuo, es el actor de la entrega de experiencia y su papel activo lo convierte en un facilitador del desarrollo social.

Esta inmersión de la experiencia con el contacto personal, es algo que sucede en un preciso momento que queda grabado en la memoria, cuyo común denominador está relacionado con el contacto con otras personas y la autenticidad (Arnould \& Price, 1993; Chandralal \& Valenzuela, 2013). 
La investigación de Chandralal y Valenzuela (2013), revela en sus entrevistas en profundidad, nueve aspectos principales relacionados a la experiencia memorable, las ocho primeras de corte cognitivo y la última afectiva, todas relacionadas con la memoria.

1. Significado percibido

2. Oportunidades percibidas para encontrar experiencias locales auténticas

3. Importancia percibida

4. Novedad percibida

5. Oportunidades percibidas para interacciones sociales

6. Hospitalidad local percibida

7. Serendipia y sorpresas

8. Profesionalismo percibido de las guías locales

9. Emociones positivas

El contacto humano, el sentido de novedad, la sorpresa y el significado de lo percibido, añaden a la experiencia un componente poco explorado en otros estudios y que se relaciona con los puntos 5 y 6 del estudio de Chandralal y Valenzuela. De este modo, encontramos que las interacciones sociales y la hospitalidad local tienen un vínculo con el contacto humano, por lo tanto, con capacidad de generar relaciones que inspiran autenticidad y significado a la experiencia.

Chandralal y Valenzuela (2013) traducen de manera menos compleja aquellos factores que involucran una experiencia desde el campo afectivo y cognitivo, en las entrevistas de profundidad la percepción de los sentidos juega un rol importante en la memoria.

Es así como encontramos que las experiencias memorables son el resultado de aspectos cognitivos, afectivos y de comportamiento, que permanecen más tiempo grabados en la memoria como consecuencia de percibir e interactuar con la misma experiencia. 


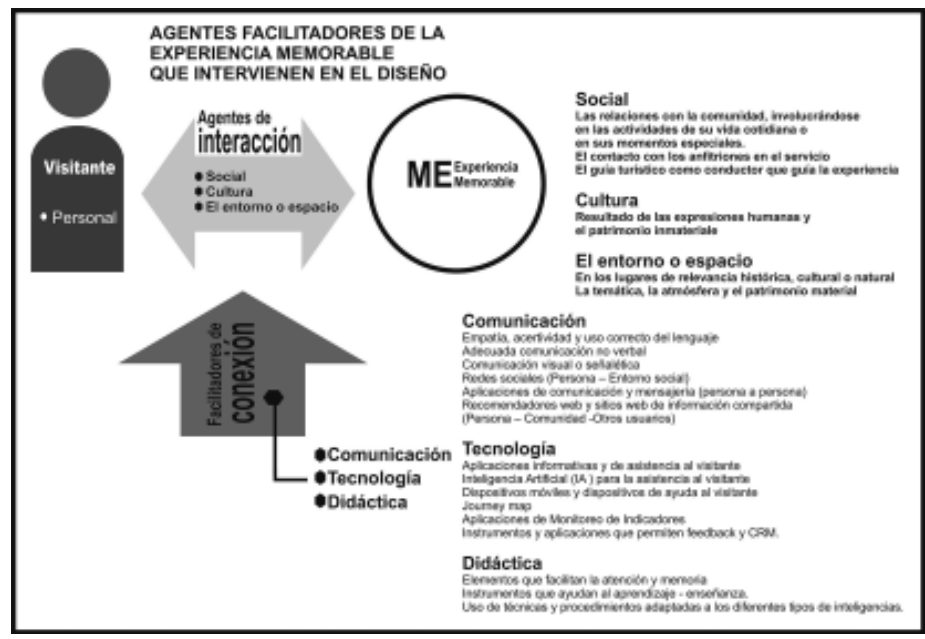

Figura 4. Agentes facilitadores de la experiencia memorable que intervienen en el diseño.

La sola conjunción de elementos no garantiza una experiencia memorable, está sujeta a la presencia de facilitadores que posibiliten una conexión, que actúen directamente en tres campos principales: la comunicación, la tecnología y la didáctica, como vía del aprendizaje-enseñanza.

Por otro lado, la didáctica en el diseño nos orienta a establecer estrategias para comunicar y otorgar un sentido a la experiencia, aprovechando los elementos personales, sociales y el entorno. Una de las características de la didáctica es su configuración histórico-social. Se refiere a que el enseñar y el aprender ha sido connatural al hombre desde su existencia y que el aprendizaje tiene una importante dimensión social porque aprendemos en la relación con los demás, y para integrarnos eficaz y creativamente en la sociedad (Hinojosa, Gallego, \& Moreno, 2011).

En efecto, los procesos didácticos, de acuerdo con Hinojosa et al. (2011, p. 28), tienen por finalidad la consecución de los objetivos educativos establecidos en las diferentes áreas de conocimiento definidos en términos de capacidades (cognitivas, afectivas, motrices, de relación e integración social) que integrados a un diseño de experiencia del visitante adquieren una connotación especial y mayores posibilidades de retenerse en la memoria como resultado de una experiencia memorable (Figura 5). 


\begin{tabular}{|c|l|}
\hline \multicolumn{1}{|c|}{ Autores } & \multicolumn{1}{c|}{ Definiciones } \\
\hline Zabalza, M. A. (1990) & $\begin{array}{l}\text { Campo de conocimientos, de investigaciones, de propuestas teóricas y prácticas que se } \\
\text { centran sobre todo en los procesos de enseñanza-aprendizaje. }\end{array}$ \\
\hline Torres, S. De La (1993) & $\begin{array}{l}\text { Disciplina pedagógica que se ocupa de los procesos de formación en contextos } \\
\text { deliberantemente organizados. }\end{array}$ \\
\hline Martín Molero, F. (1999) & $\begin{array}{l}\text { Ciencia aplicada al proceso de enseñanza-aprendizaje con vistas al crecimiento } \\
\text { intelectual y humano del sujeto mediante la optimización de dicho proceso. }\end{array}$ \\
\hline Gervilla, A. (2000) & $\begin{array}{l}\text { Ciencia de la educación que estudia todo lo relacionado con la enseñanza: diseño de } \\
\text { las mejores condiciones, ambiente, clima, para conseguir un aprendizaje excepcional y } \\
\text { el desarrollo completo del alumno. }\end{array}$ \\
\hline Medina Rivilla, A. (2002) & $\begin{array}{l}\text { Disciplina o tratado riguroso de estudio y fundamentación de la actividad de enseñanza } \\
\text { en cuanto que propicia el aprendizaje formativo de los estudiantes en los mas diversos } \\
\text { contextos. }\end{array}$ \\
\hline Sevillano, M. L. (2004) & $\begin{array}{l}\text { Ciencia teórico-normativa que guía de forma intencional el proceso optimizador de la } \\
\text { enseñanza-aprendizaje en un contexto determinado e interactivo, posibilitando la } \\
\text { aprehensión de la cultura con el fin de conseguir el desarrollo integral del estudiante. }\end{array}$ \\
\hline González, F. E. (2008) & \begin{tabular}{l} 
Acción intencionada de comunicar conocimientos que conduce a la educación. \\
\hline
\end{tabular}
\end{tabular}

Figura 5. Definición de didáctica. Adaptado de Hinojosa et al., 2011, p. 16.

\section{Conclusiones}

Las experiencias memorables se producen en diferentes espacios o momentos durante la visita turística, por ello, es preciso indicar que una planificación adecuada del recorrido de la experiencia del cliente, puede advertir o generar las oportunidades requeridas para garantizar una experiencia memorable.

Las experiencias memorables son el resultado de aspectos cognitivos, afectivos y de comportamiento, que permanecen más tiempo grabados en la memoria como consecuencia de percibir e interactuar con la misma experiencia.

El contacto del visitante con personas y comunidades favorece el desarrollo de experiencias memorables. Son las personas: guías turísticos, personal de servicio o los mismos anfitriones, quienes actúan como facilitadores o conductores de la experiencia.

El visitante logra introducirse en la experiencia gracias a estos facilitadores que permiten ver el lado íntimo del lugar visitado, interpretarlo y hacerlo suyo. En ese sentido, el contacto humano le otorga autenticidad y significado a la 
experiencia turística. Sin embargo, esto no ocurre automáticamente, el visitante debe lograr aquello que algunos autores llaman inmersión o estado de mindfulness, posición que le brinda un soporte especial a la experiencia, una apertura de conciencia que permite al hilo conductor entrar en el consciente del visitante.

Tal como sucede en la vida cotidiana, los encuentros que nos transforman y marcan nuestra memoria suceden de manera inesperada, los identificamos en momentos que luego se convierten en especiales y están cargados de imágenes, rostros y una conjunción de diversas percepciones que quedan en nuestra memoria para dar sentido o describir lo experimentado, ison los momentos memorables de la vida aquellos que el viajero busca encontrar? Definitivamente no podemos esperar que todo sea espectacular o memorable en la vida, pero es, ese tipo de expectativa que buscamos encontrar en una experiencia de viaje memorable.

Arnould y Price (1993) trabajan las experiencias extraordinarias en el aspecto personal, y la relación con los demás, añadiendo una especial connotación a la relación con la naturaleza. Una experiencia memorable en todo sentido involucra el espacio donde se desenvuelve y este adquiere sentido cuando la relación con los demás es capaz de provocar alguna reacción en el interior de la persona, de índole cognitivo y afectivo. El tiempo, el espacio y la persona configuran este preciso momento memorable, para hacerlo más razonable y descriptible. Las emociones se traducen en frases y reconocimientos de percepciones que también terminan perdurando en la memoria.

El servicio es percibido por el cliente como un todo y los puntos de contacto son el nexo entre el cliente y el servicio. Bajo esta mirada las investigaciones han buscado respuestas en base a la deducción de preguntas generales referidas a la satisfacción para advertir factores que se relacionan con la memoria, agrupándolos en afectivos, cognitivos y de comportamiento, por un lado aquellas características del servicio y por el otro la aguda desagregación de cada parte del servicio han permitido encontrar los factores y dimensiones que influyen en la experiencia memorable. 
Las evaluaciones o conjunto de percepciones experimentadas en un servicio juegan una suerte de operación en la mente del visitante, una operación que implica la valoración de lo percibido y un resultado relacionado a su aprobación o conformidad. Entonces iqué sucede cuando el balance es positivo, o encontramos aspectos no percibidos o negativos percibidos? Se produce un efecto de compensación en la que el valor de los aspectos percibidos supera aquellos negativos.

De acuerdo con las investigaciones de Arnould y Price (1993) una experiencia se convierte en memorable cuando se involucra el plano personal, el plano social y el entorno o medio donde se desenvuelve. En este sentido identificamos que la experiencia memorable del visitante se desenvuelve en la intersección de tres planos principales que se conectan entre sí y permiten interacción: el primero identificado como plano personal en donde la memoria, los recuerdos, y los sentidos juegan un papel importante al momento de hacer razonables las percepciones, añadiendo argumentos y elementos de juicio para obtener una reflexión en el plano personal. La segunda referida a la interacción que se desarrolla en el plano social y está compuesta por las relaciones directas entre personas, entre la comunidad, los prestadores de servicio, los guías e inclusive con aquellas en las que se emplean medios de comunicación a distancia, dándole un sentido de unidad a la experiencia bajo una dimensión humana.

Las interacciones sociales se involucran con la cultura, como conductor de las expresiones humanas, en este ámbito el patrimonio inmaterial y aquellos elementos no tangibles de la experiencia con dimensión humana involucran el plano social de una forma muy especial descrita por Arnould y Price (1993) como communitas o comunión.

El entorno o medioambiente, en donde se desarrolla la experiencia, representa el espacio físico y sus elementos, los mismos que configuran una atmósfera o temática, como elemento motivador que dirigen el comportamiento y la actitud frente a la experiencia, representan el elemento tangible formando parte de las imágenes y percepciones que recibe el visitante.

En el contexto de la comunicación es importante aplicar un especial cuidado en la empatía, la asertividad, uso adecuado del lenguaje y lenguaje no verbal, como el pilar de la atención al visitante y la entrega de valor en la 
experiencia. Otro facilitador es la comunicación visual y la señalética como soporte de la experiencia turística, del mismo modo las diversas formas de comunicación bajo la conectividad del internet son importantes por el valor que otorgan las opiniones e interacciones que fortalecen vínculos con los participantes.

Como consecuencia de la conectividad la tecnología está al alcance del usuario, es decir, el turista de hoy es un usuario omnicanal, movilizándose en diferentes soportes como el smarthphone, la tablet, laptop o cualquier dispositivo conectado que le permite acceder a la nube. En este tipo de aplicaciones y plataformas de contenido el visitante logra nutrirse de conocimiento y se asiste por medios inteligentes. Debido a esta interacción, se genera una gran cantidad de datos, por lo tanto, es necesario contar con un sistema de control y organización, en tal sentido se sugiere utilizar herramientas como el CRM customer relationship management, como una solución que organiza y simplifica la gestión con el cliente o en su defecto, sistemas y plataformas con conectividad en la nube basadas en el Journey map.

\section{Referencias}

Ali, F., Hussain, K., \& Ragavan, N. A. (2014). Memorable customer experience: Examining the effects of customers experience on memories and loyalty in Malaysian resort hotels. Procedia-Social and Behavioral Sciences, 144, 273-279. Recuperado de https://doi.org/10.1016/j.sbspro.2014.07.296

Arnould, E. J., \& Price, L. L. (1993). River magic: Extraordinary experience and the extended service encounter. Journal of consumer Research, 20(1), 24-45. Recuperado de https://doi.org/10.1086/209331

Ballantyne, R., Packer, J., \& Sutherland, L. A. (2011). Visitors' memories of wildlife tourism: Implications for the design of powerful interpretive experiences. Tourism Management, 32(4), 770-779. Recuperado de https:/l doi.org/10.1016/j.tourman.2010.06.012

Brent, J. R., Tung, V., \& Ritchie, R. J. (2011). Tourism experience management research: Emergence, evolution and future directions. International Journal of Contemporary Hospitality Management, 23(4), 419-438. Recuperado de http://dx.doi.org/10.1108/09596111111129968

Brugnoli, G. (2009). Connecting the dots of user experience. Journal of Information Architecture, 1(1). Recuperado de https://core.ac.uk/download/pdf/55208044.pdf

Carballo, R. R., Moreno, S., León, C., \& Brent, J. R. (2015). La creación y promoción de experiencias en un destino turístico. Un análisis de la investigación y necesidades de actuación. Recuperado de https://doi.org/ 10.6018/turismo.35.221511

Chandralal, L., \& Valenzuela, F. R. (2013). Exploring memorable tourism experiences: Antecedents and behavioural outcomes. Journal of Economics, Business and Management, 1(2), 177-181. doi: 10.7763/JOEBM.2013.V1.38

Grönroos, C. (1990). Service Management and Marketing: Managing the Moments of Truth in Service Competition. Lexington: Lexington Books. 
Hinojosa, R. N., Gallego, M. R. R., \& Moreno, M. B. (2011). Didáctica y currículum para el desarrollo profesional docente. Recuperado de https://www.researchgate.net/publication/268810901_Didactica_y_ curriculum_para_el_desarrollo_profesional_docente

Horváth, Z. (2013). Memorable tourism experience. The Routledge handbook of cultural tourism, 375. Recuperado de https://eprints.usq.edu.au/24290/3/Horvath_CTH_2011_AV.pdf

Hosany, S., \& Witham, M. (2010). Dimensions of cruisers' experiences, satisfaction, and intention to recommend. Journal of Travel Research, 49(3), 351-364. Recuperado de https://doi.org/10.1177/0047287509346859

Kabat-Zinn, J. (2003). Mindfulness based interventions in context: past, present, and future. Clinical psychology: Science and practice, 10(2), 144-156. Recuperado de https://doi.org/10.1093/clipsy.bpg016

Kim, J. H., Ritchie, J. B., \& McCormick, B. (2012). Development of a scale to measure memorable tourism experiences. Journal of Travel Research, 51(1), 12-25. Recuperado de https://doi.org/10.1177/ 0047287510385467

Lawson, M. (2018). Finding the right time: How leading marketers redefine moments of assistance. think with google. Recuperado de https://www.thinkwithgoogle.com/int//en-cee/insights-trends/research-data/finding-righttime-how-leading-marketers-redefine-moments-assistance/

Maloney, D. (2017). Why Domino's delivers more than 15 ways to order pizza. Recuperado de http://n9.cl/Pzfc

Moreno, A. M., Los Santos, I. S., \& Pascual, J. A. V. (2017). Propuesta de modelo teórico para el análisis del impacto de los factores culturales y de personalidad en la demanda de servicios turísticos. Esic market, 157, 275-313. doi: 10.7200/esicm.157.0482.2e

Pine, B. J., \& Gilmore, J. H. (1998). Welcome to the experience economy. Harvard business review, 76, 97-105. Recuperado de https://www.ncbi.nlm.nih.gov/pubmed/10181589

Richardson, A. (2010a). Using customer journey maps to improve customer experience. Harvard Business Review, 15(1), 2-5. Recuperado de https://hbr.org/2010/11/using-customer-journey-maps-to

Richardson, A. (2010b). Understanding Customer Experience. Recuperado de https://doi.org/10.1509/jm.15.0420

Stickdorn, M., \& Zehrer, A. (2009, November). Service design in tourism: Customer experience driven destination management. In First Nordic conference on service design and service innovation, Oslo (pp. 1-16). Recuperado de http://citeseerx.ist.psu.edu/viewdoc/summary? doi=10.1.1.424.845

Tung, V. W. S., \& Ritchie, J. B. (2011). Exploring the essence of memorable tourism experiences. Annals of tourism research, 38(4), 1367-1386. doi:10.1016/j.annals.2011.03.009

Verhoef, P. C., Lemon, K. N., Parasuraman, A., Roggeveen, A., Tsiros, M., \& Schlesinger, L. A. (2009). Customer experience creation: Determinants, dynamics and management strategies. Journal of retailing, 85(1), 31-41. doi:10.1016/j.jretai.2008.11.001

Zehrer, A. (2009). Service experience and service design - concepts and application in tourism SMEs, Managing Service Quality. doi: 10.1108/09604520910955339 
